\title{
Anlegerschutz im System des Kapitalmarktrechts Rechtsgrundlagen und Ausblicke
}

\author{
THOMAS M.J.MÖLLERS
}

\begin{abstract}
Einleitung
Nicht von ungefähr ist das Kapitalmarktrecht, so wie wir es heute kennen, auch einer Finanzkrise geschuldet - nach dem Schwarzen Freitag vom 24. Oktober 1929 war es Roosevelt, der mit dem SA und SEA die Grundlagen für das US-amerikanische Kapitalmarktrecht legte, dessen wichtigste Instrumentarien dann weltweit, auch in Europa in den 70er Jahren des letzten Jahrhunderts übernommen wurden. Das US-amerikanische Recht ist nicht für seine systematische Durchdringung bekannt, sondern Gesetze werden oft ad-hoc als Reaktion auf Krisen entwickelt,' Dabei zeichnet es sich durch eine Reihe von Besonderheiten aus, etwa den Umstand, dass die Kompetenz für das Kapitalmarktrecht beim Bund liegt, während das Gesellschaftsrecht in der Kompetenz der US-amerikanischen Bundesstaaten verortet ist. $\mathrm{Zu}$ den Besonderheiten gehört auch der im Vergleich zu Europa generell liberalere Ansatz, Probleme gerne dem Markt zu überlassen, da dieser eher Lösungen parat habe als der Gesetzgeber als Regelungsgeber.

In Europa hat man seit mehr als 100 Jahren in den Mitgliedstaaten ein Gesellschaftsrecht, das relativ ausgereift ist, ${ }^{2}$ aber nur ein recht junges Kapitalmarktrecht, das erst gut 20 Jahre alt ist ${ }^{3}$ und infolgedessen nur an einigen Universitäten unterrichtet wird- Gleichwohl sind inzwischen Tausende von Vorschriften entstanden, die nur noch schwer zu überschauen sind. ${ }^{4}$

Neben der Einführung des Securities Act 1933 (SA) und Securities Exchange Act 1934 (SEA) durch Roosevelt bildete auch der Sarbanes-Oxley Act (SOX) ein solches Gesetz; zu den Finanzkrisen im Allgemeinen s, Cox/Hillman/Langevoort Securities Regulation ${ }^{5}$, 2006, Chap. 1; Reinhart/Rogoff This Time is Different, Eight Centuries of Financial Folly, 2009; zum Sarbanes-Oxley Act (SOX) Möllers 4 E.C.ER. (2007) 173 ff

Zur Geschichte des Gesellschaftsrechts s. Schubert/Hommelhoff (Hrsg.) Hundert Jahre modernes Aktienrecht, 1985 sowie dies. Die Aktienrechtsreform am Ende der Weimarer Republik, 1987.

S. etwa Hopt in Grundmann (Hrsg.), Systembildung und Systemlücken in Kerngebieten des Europäischen Privatrechts, 1999, S. 307, 311 ff.; zur Entwicklung des ungleich älteren Börsenrechts s. Merkt in Hopt/Rudolph/Baum Börsenreform, 1997, S. 17 ff.

Für eine Übersicht s. die Datenbank bei www.thomas-moellers.de. Zur jeweils dreistufigen Gliederungsstruktur auf nationaler und europäischer Ebene s. Möllers ZE u P 2008,480 ff.; den. in Gsell/Herresthal (Hrsg.), Vollharmonisierung im Privatrecht, 2009, S. 247, 253.
\end{abstract}


Die Finanzkrise hat jüngst die „Fragilität" der Finanzmärkte verdeutlicht. Auf der Ebene der G 20 versucht man seit Monaten, Schwachstellen zu erkennen und darauf zu reagieren. ${ }^{5} \mathrm{Neu}$ ist dabei, dass man ernstlich gewillt ist, auf internationaler Ebene einheitliche Regelungen zu entwickeln. Klaus J. Hopt kann ganz unstrittig als „Vater" des deutschen, ja wohl des europäischen Kapitalmarkt rechts genannt werden. Mit unzähligen Beiträgen hat er die Grundlagen des Kapitalmarktrechts gelegt ${ }^{6}$ und Schwachstellen thematisiert und dabei nicht nur das bisherige Recht aufbereitet, sondern „,vorgedacht" ${ }^{7}$ und den Weg für künftige Entwicklungen aufgezeigt. ${ }^{8}$

Der vorliegende Beitrag möchte skizzenartig einige Schwachstellen des jetzigen Kapitalmarktrechts verdeutlichen, um damit den weiteren Handlungsbedarf zu verdeutlichen. Dabei mag es helfen, wenn man stärker als bisher üblich versucht, das Kapitalmarktrecht zu strukturieren. Im Einzelnen soll zwischen Marktteilnehmern (L), den relevanten Finanzdienstleistungen der Finanzintermediäre (IL) sowie den Finanzprodukten und Informationen börsennotierter Unternehmen (HL) differenziert werden. Schließlich ist auf die Umsetzung und Rechtsdurchsetzung (IV.) sowie auf die juristische Methodik im Kapitalmarktrecht einzugehen (V). Dabei sollen neben wichtigen positiven Entwicklungen vor allem Lücken im Anlcgerschutz angesprochen werden. Einzelne Probleme bleiben einer vertieften Analyse vorbehalten.

\section{Marktteilnehmer}

\section{$h \quad$ Private und professionelle Investoren}

Im Bank- und Kapitalmarktrecht stehen börsennotierte Unternehmen professionellen Anlegern und Kleinanlegern gegenüber. Als dritte Gruppe fassen sog. Finanzintermediäre Informationen über börsennotierte Unternehmen zusammen und bewerten diese. Die MiFID hat eine Unterscheidung in professionelle und private Investoren getroffen. Für professionelle Investoren sind zahlreiche Informationspflichten reduziert.' Das ist überzeugend, weil ansonsten Transaktionskosten entstünden, die der kundigen Seite pater-

s. etwa London Summit 2009; Pittsburgh Suinmit 2009; St. Andrews Summit 2009; Nachweise finden sich bei www.thomas-moellers.de.

- Wegbereitend Hopt Der Kapitalanlegerschutz im Recht der Banken 1975; den. Gutachten G zum 51. DJT 1976, S. 1 ff.; ders. ZHR 140 (1976) 201; ZHR 141 (1977) 389; jüngst ders. WM 2009,1873.

Zur Unterscheidung s. Lerche BayVBL 2002,649, 650; Möllers Juristische Arbeitstechnik und wissenschaftliches Arbeiten, 2010, Rn. 297.

s Exemplarisch etwa Hopt Z H R 159 (1995) 135; Hopt/Voigt (Hrsg.), Prospekt- und Kapitalmarktinformationshaftung, 2005 sowie gerade Fn* 3,6.

'S. Art. 4 Abs. 1 Nr. 11 i.V.m. Anh. II der MiFID; umgesetzt in $\S \S 31$ Abs. 9,31 a Abs. 2 WpHG. 
nalistisch aufgedrängt würden. ${ }^{10}$ Allein: Die Finanzkrise zeigt, dass selbst vermeintlich professionelle Landesbanken nicht professionell genug waren, um die Risiken von Finanzprodukten richtig einzuschätzen. Auch zahlreiche Kämmerer von Kommunen klagen wegen unzureichender Aufklärung," Juristisch sind auch sie professionelle Anleger, so dass ihnen gegenüber die Pflichten des $\S 31 \mathrm{WpHG}$ weitgehend nicht gelten. ${ }^{12}$

\section{Große und kleinere börsennotierte Unternehmen}

Europäisches Kapitalmarktrecht ist weiterhin lückenhaft. So gelten zahlreiche Informationspflichten nur für den geregelten Markt, nicht für den Freiverkehr, wie den Entry Standard der Frankfurter Börse oder den Freiverkehr an den anderen regionalen Börsen. Die Größe der börsennotierten Unternehmen wird plastisch in den verschiedenen „Marktsegmenten" der Börsen wiedergegeben, etwa dem DAX, MDAX, SDAX und dem Prime Standard der Frankfurter Börse, Die drei großen Börsen in Europa werben um kleine Unternehmen. Der Freiverkehr hat für die Unternehmen den Vorteil, dass sie dort nicht den strengen Publizitätspflichten des geregelten Marktes unterliegen und die damit verbundenen Kosten sparen. Ansonsten bestehen für kleine börsennotierte Unternehmen aber viele Nachteile am Kapitalmarkt: Sie können sich keine großen Investor Relations-Abteilungen leisten, welche die Web-Seite des Unternehmens pflegen und Finanzanalysten, Fonds und andere Anleger regelmäßig mit Informationen versorgen. Zudem erstellen die großen Wertpapierdienstleistungsunternehmen nur selten Finanzanalysen über kleine börsennotierte Unternehmen. Schließlich werden die Wertpapiere solcher Unternehmen deutlich weniger gehandelt.

Geringe Handelsumsätze laden zu Kursmanipulationen ein. ${ }^{13}$ Auch durch Spam-Mails werden nicht selten Kleinanleger wahrlich „abgezockt". Hier herrschen noch teilweise Wildwest-Manieren, die dem Kapitalmarkt als Institution wenig zuträglich sind. Der potentiell höheren Gefahr von Insiderhandel und Marktmanipulationen bei den Werten kleinerer börsenno-

10 S, auch Leisch Informationspflichten nach $§ 31$ WpHG, 2004, S. 163 ff.

" Vor allem die Deutsche Bank sieht sich zahlreichen Klagen ausgesetzt, s, etwa O L G Naumburg v. 24.3.2005, WM 2005, 1313; OLG Bamberg v. 22.5.2009, WM 2009, 1082; OL G Celle v. 30.9.2009, BB 2009,2265 . Im wohl umfangreichsten Streit dieser An (SpreadLadder-Swaps und einer Schadensersatzklage in Höhe von 50 Mio. Euro) wird das O L G Düsseldorf voraussichtlich am 18.12.2009 über eine Berufung der zuvor vor dem Landgericht Wuppertal (v. 16.7.2008, ZIP 2008,2014$)$ unterlegenen Stadt Hagen entscheiden; zu Saint-Etienne s. F A 2 v. 1.11.2009, S. 19.

12 S. Begr. RegE FRUG; BT-Drucks. 16/4028, S. 66; Koller in Assmann/Schneider, WpHG 2009', \% 31a Rn. 3; kritisch Bracht WM 2008,1386,1388,

${ }^{13}$ Fleischer ZBB 2008, 137. 
tierter Unternehmen, die durch die geringere Marktkapitalisierung hervorgerufen wird, wird rechtlich noch nicht angemessen begegnet. Während bisher eher die großen Marktsegmente kontrolliert und überwacht wurden, müsste künftig wohl eine deutlich verbesserte Aufsicht durch die Börsen und die staatliche Aufsicht, also die Bundesanstalt für Finanzdienstleistungsaufsicht (BaFin), auch über die Werte kleinerer börsennotierter Unternehmen stattfinden. ${ }^{14}$

\section{Finanzintermediäre}

Anleger vertrauen auf die Sachkunde von Finanzintermediären wie Rating $\neg$ Agenturen, Finanzanalysten und Wertpapierdienstleistungsunternehmen und greifen auf deren Informationen zurück. Aufklärungspflichten bilden immer noch das dominante Instrumentarium des Kapitalmarktrechts. Gegenüber dem Verbot haben sie den Vorteil, dass das Geschäft erlaubt bleibt und „nur" gegebenenfalls über die Risiken aufzuklären ist. Überraschenderweise wird in den einschlägigen Lehrbüchern nicht auch die Vermeidung von Interessenkonflikten als allgemeines Instrumentarium des Kapitalmarktrechts herausgearbeitet. ${ }^{15}$ Dabei ist die Vermeidung oder zumindest die Aufklärung über Interessenkonflikte nicht nur eine Pflicht der Banken gegenüber ihren Kunden ( 31 Abs. 1 Nr. 2 WpHG), organisatorisch im Rahmen der Compliance festgelegt ( $\$ 33$ Abs. 1 Nr. $3 \mathrm{WpHG}$ ), sondern begründet auch eine Pflichtenstellung von Finanzanalysten ${ }^{16}$, Rating-Agenturen ${ }^{17}$ oder Wirtschaftsprüfern. ${ }^{18}$ Dieser Ansatz ließe sich noch erheblich weiter ausbauen: irreführende Disclaimer sind zu verbieten; ${ }^{19}$ Verletzungen sollten auch zivilrechtlich sanktioniert werden.

Laut Auskunft des zuständigen Referenten nimmt sich die BaFin dieser Problematik in letzter Zeit auch besonders an. Dieser erhöhte Verfoigungsdruck scheint sich schon in einer Reduzierung der einschlägigen Delikte niederzuschlagen, s. auch $\mathrm{Ä}^{\wedge}$ firc-Jahresbericht $2008, \mathrm{~S}, 156$ ff. Zu Vorschlägen s. auch Hopt/Baum in Hopt/Rudolph/Baum, Börsenreform, $1997,5.287,372$ ff.

is Nicht angesprochen etwa von Buck-Heeb Kapitalmarktrecht ${ }^{3}$, 2009; Claussen Banken- und Börsenrecht', 2008; Kümpel/Veil Wertpapierhandelsgesetz ${ }^{4}, 2006$.

${ }^{16}$ § 34b Abs. 1 Nr. 2 WpHG; hierzu Möllers, AfP 2010 (Heft 2).

"Art. 5 Vorschlag für eine Verordnung des Europäischen Parlaments und des Rates über Rating-Agenturen v. 12,1 1.2008, KOM (2008) 704 endg.; hierzu ausführlich Möllen JZ $2009,861$.

18 Art.22 Abs. 2 Änderungs-RiL $2006 / 43$ / E Gv, 17,5.2006 über die Abschlussprüfungen von Jahresabschlüssen und konsolidierten Abschlüssen, ABI. Nr. L 157, S. 87.

19 Zu einem solchen Disclaimer s. etwa Möllers/'HolznerNZG $2008,166,171$. 


\section{IL Finanzdienstleistungen der Finanzintermediäre}

\section{Ausgewählte Missstände bei Finanzintermediären}

a) Rating-Agenturen

Bis heute gibt es in diesem Bereich noch deutliche Defizite. Rating-Agenturen gaben viel zu gute Ratings über strukturierte Finanzprodukte (Collateralised Debt Obligations - CDO) ab und hatten umfangreiche Interessenkonflikte, weil sie etwa diese Produkte entwickelten, um sie dann mit einem positiven Rating zu bewerten. ${ }^{20}$

\section{b) Finanzanalysten und Journalisten}

Anders als in den USA stehen in Deutschland Kleinanlegern in der Regel Finanzanalysen nicht zur Verfügung. Sie sind auf Zusammenfassungen durch Dritte, z.B. Journalisten, angewiesen. Inhaltlich müssen Finanzanalysen und Zusammenfassungen etwa den Ersteller nennen und auf Interessenkonflikte hinweisen. Hiergegen verstoßen Journalisten in Deutschland noch regelmäßig. ${ }^{21}$

\section{c) Wertpapierdienstleistungsunternehmen}

Für den Anleger positiv ist sicherlich, dass Wertpapierdienstleistungsunternehmen aufgrund europäischen Rechts verpflichtet wurden, sich vor einer Anlageberatung mit den Wünschen und der finanziellen Situation des Anlegers intensiv zu beschäftigen („know your customer"). Für den Anleger positiv ist auch, dass nun die Anlageberatung in einem Beratungsprotokoll schriftlich festzuhalten ist, § 34 Abs. 2a S, 3-5 WpHG. ${ }^{22}$ Zweifelhaft bleibt aber, ob dem deutschen Gesetzgeber überhaupt die Kompetenz zusteht, eine derartige Aufzeichnungspflicht zu schaffen. ${ }^{23}$ Daneben existieren zahlreiche weitere ungeklärte Einzelfragen: Die Banken/Finanzdienstleister dürfen nach

20 Hierzu Möllers 4 CMLJ 2009, 477; übersetzt als ders. JZ 2009, 861.

${ }^{21}$ Zustimmend auch Möllers AfP 2010 (im Erscheinen); ders. in KK-W p HG, 2007, § 34b Rn. 234, ihm folgend Koller in Assmann/Schneider, WpHG 2009, § 34b Rn. 73; Corres m Habersack/Mülbert/Schlitt, Handbuch Kapitalmarktinformation, 2008, § 24 Rn. 176; s. auch Jahn FAZ v. 4.11.2009, S. 21.

${ }^{22}$ Vergleichbare Pflichten gab es bisher schon mit $§ \S 6$ Abs. 1 S. 2,61 Abs. 1 S. 2 W G Allerdings sind diese deutlich weniger umfangreich, s. Böhm B KR 2009, 221, 224.

${ }^{23}$ Kritisch Möllers/Wenninger Stellungnahme im Rechtsausschuss als Gutachter des Deutschen Bundestages zum Regierungsentwurf eines Gesetzes zur Neuregelung der Rechtsverhältnisse bei Schuldverschreibungen aus Gesamtemissionen und zur verbesserten Durchsetzbarkeit von Ansprüchen von Anlegern aus Falschberatung (BT-Drs. 16/12814) - 29.4,2009, abrufbar unter www.thomas-moellers.de; s. auch Möllers in Gsell/Herresthal $\left({ }^{* *{ }^{n}} \mathrm{~g}^{\prime}\right)>$ Vollharmonisierung im Privatrecht, 2009, S. 247; Böhm aaO 228; aA Leuering/ Zetsche NJ\V2009,2856, 2859. 
Abschluss der Beratung die Transaktion erst durchführen, wenn der Kunde das Beratungsprotokoll erhalten hat. Gerade in der Anlageberatung spielt der zeitliche Faktor aber eine erhebliche Rolle, Die Erstellung und Ubermittelung eines solchen Protokolls kann schlicht zu lange dauern: Wer haftet für die sich in der Zwischenzeit geänderte Marktkonditionen? Darüber hinaus birgt die Beweislastumkehr zu Ungunsten der Banken bei einer möglichen Anfechtung des Beratungsprotokolls erhebliche Risiken für die Bank, die gar zum Missbrauch führen können. Der Kunde hat nach Eingang des Protokolls eine Woche Zeit, dasselbige anzufechten. Da die Bank die Beweiskst trifft, könnte ein Kunde bequem bei einem eventuell auftretenden Kursverlust innerhalb dieser Woche das Protokoll anfechten. Die Bank müsste dann mühselig den Gegenbeweis bringen, dass das Protokoll weder unvollständig noch inkorrekt ist. Auch sollte für den Kunden die Möglichkeit bestehen, auf das Beratungsprotokoll zu verzichten. ${ }^{24}$ In diesen Fällen schießt der Anlegerschutz über das Ziel hinaus und führt zu einer unangemessenen Benachteiligung der Banken.

Darüber hinaus sind bei Banken und Wertpapierdienstleistungsunternehmen zahlreiche Dienstleistungen der Optimierung zugänglich. Zwar verlangt das Gesetz, dass Information und Beratung, mit der erforderlichen Sach-

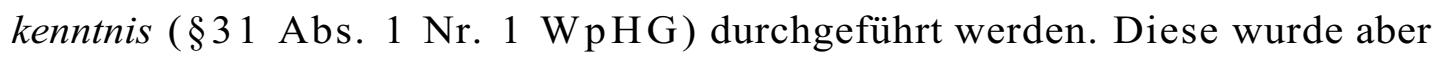
nicht weiter gesetzlich konkretisiert. ${ }^{25}$ Faktisch sind die 16- bis 30 -jährigen Bankmitarbeiter oft überfordert, dem Anleger kundengerechte Anlage vorschlage zu unterbreiten. ${ }^{26}$

Die MiFID konkretisiert die Finanzdienstleistungen und schafft damit Rechtsklarheit. Zulasten des Anlegerschutzes bedeutet sie aber auch einen Rückschritt ${ }^{\wedge}$ weil etwa ausdrücklich Discount-Broker oder standardisierte Aufklärung zugelassen werden. ${ }^{27}$ In der Praxis existieren Mischformen zwischen Anlageberatung und Vermögensverwaltung. Dazu gehören die sog. graue Vermögensverwaltung und Zweitberatung. Unklar ist, in welchem Umfang solche Handlungsformen einer gerichtlichen Kontrolle zugänglich sind..$^{28}$

Zu dieser Forderung Böhm a aO 222.

25 Im Zivilrecht ist aber eine umfangreiche Recherchepflicht anerkannt, s. B GH v. 6.7 1993, BGHZ 123,126,128 f. - Bond; Möllers in KK-WpHG, 2007, § 31 Rn. 68 ff.; Fuchs in Fuchs, WpHG, 2009 , § 31 Rdn. 25 ff.

${ }^{26}$ Kritisch etwa Focus 26/2009 v. 22.6.2009, S. 96 ff. Deutlich auch Leuering/Zeische NJW 2009, 2856,2861: Die Filialberaterwerden von Auswertungs- und Vertriebszentralen fremdgesteuert und haben keinen Zugang zu Finanzexperten.

${ }^{17}$ Hierzu Möllers in KK-WpHG, 2007, § 31 Rn. 195 ff., 243 f.; angedeutet auch bei Fuchs in Fuchs, WpHG, 2009, vor $\S$ 3-37a Rn. 70.

${ }^{28}$ Möllers WM 2008, 93; s. auch ders. in Gsell/Herresthal (Hrsg.), Vollharmonisierung im Privatrecht, 2009, S. 247,265; ihm folgend Fuchs in Fuchs, WpHG, 2009, § 31 Rn. 245 f. 
Zudem existieren Interessenkonfliktel weil Banken üblicherweise hauseigene Finanzprodukte anpreisen. Die Rechtsprechung zum kick-back ${ }^{29}$ betrifft nur einen kleinen Teilaspekt. Obwohl der BGH die Vermeidung von Interessenkonflikten als allgemeinen Grundsatz postuliert, ${ }^{30}$ hat er es nicht beanstandet, wenn nicht darüber aufgeklärt wird, dass nur hauseigene Produkte empfohlen werden, ${ }^{31}$ Diese Rechtsprechung widerspricht dem Leitbild, Finanzdienstleister dazu zu verpflichten, im bestmöglichen Interesse ihrer Kunden zu handeln $(\S 31 \mathrm{WpHG}) .^{32}$ Der Markt an sog, Honorarberatung und damit Beratungsmaklern, die ohne Interessenkonflikte neutral über verschiedene Anlageformen informieren, ist noch außerordentlich gering, ${ }^{33}$ Das Versicherungsrecht ist hier weiter: Gemä $\S 60$ Abs. 2 WG muss der Versicherungsmakler auf seine beschränkte Auswahl hinweisen. ${ }^{34}$ Vergleichbares wäre Banken auch zuzumuten.

Auch Banken schulden als Finanzintermediäre ein Minimum an Aufklärung, die etwa in Form standardisierter Aufklärung durch Broschüren stattfindet. Unzureichend dürfte es sein, wenn beim Online-Broking keine aktuellen Börsenkurse bereitgehalten werden. Der Anleger ist dann oftmals gezwungen, »bestens" zu kaufen statt eine große Spread-Spanne durch einen Stopp-Kurs begrenzen zu können.

\section{Fiduziarische Pflichten von Finanzintermediären und sonstigen Marktteilnehmern}

Es fällt auf, dass vertragliche Pflichten der Finanzintermediäre regelmäßig nur gegenüber ihren Auftraggebern, den Kunden bestehen, nicht aber gegenüber Dritten. Während Schadensersatzklagen aus Vertrag gegenüber Banken häufig auftreten, bleiben mangels Vertrags gegen Dritte Haftungslücken,

BGH v 19.12.2000, BGHZ 146，235，241 - Ender/WestLB; BGH v. 19.12.2006, BGHZ 170,226,234 - Kick Back II; BGH v. 20.1,2009, WM 2009,405,406 - Kick Back III; BGH v. 12.5.2009, WM 2009, 1274 - Kick Back IV; Möllers in KK-WpHG, 2007, § 31 Rn. 144 ff, Hopt in Baumbach/Hopt H G B ${ }^{34}, 2010$, § 347 Rn. 30.

B GH v. 20.1.2009, WM 2009, 405, 406 - Kick Back III; der Hinweis auf Möllers in KK-WpHG, 2007, § 31 Rn. 23 trifft in dieser Pauschalität nicht zu, zu Recht deshalb Haas LMK 2009, 277068.

${ }^{31}$ BGH v. 19,12,2006, ZIP 2007, 518, 520; Fuchs in Fuchs, WpHG, 2009, § 31 Rn. 79; zustimmend Schäfer/Schäfer BKR 2007, 163,164.

${ }^{32}$ Kritisch Koller ZB B 2007, 197, 198; ders. in Assmann/Schneider, W p H G ${ }^{5}, 2009$, § 31 Rn. 10; Möllers in KK-WpHG, 2007, § 31 Rn, 134.

Krohn FAZ v. 25.7.2009, S. 9; eine Ausdehnung fordernd etwa die quirin bank, s. FAZ v. 8.11.2009, 15, S. auch die Studie des BMELV v. September 2008, S. 152 (abnifbar unter http://wwv.bmelv,de/cae/servlet/contentblob/379922/publicationFile/42905/ SmdieFinanzvermittler.pdf;jsessionid=F2370B2E1310E6FCFE482F73C38955C5 ).

3* Basierend auf $\S 12$ (e)(ii), (iii)Vermittlungs-RiL 2002/92/EG v. 9.12.2002, ABI. Nr. L 9, 
wenn Finanzanalysten und Rating-Agenturen viel $\mathrm{zu}$ positive, kaum noch nachvollziehbare Empfehlungen aussprechen.

Im Gesellschaftsrecht sind die Organe der Gesellschaft gegenüber zur Treue verpflichtet. Diese fiduziarische Treuepflicht leitet sich aus der besonderen Verfügungsmacht über fremde Vermögensinteressen $a b \cdot{ }^{35}$ Dieser Gedanke trifft unmittelbar auch für die Vermögensverwaltung ${ }^{36}$ und auf Investmentfonds $^{37} \mathrm{zu}$. Ansonsten sind Bankgeschäfte als Kommissionsgeschäfte einzuordnen, welche dazu verpflichten, fremdnützig die eigenen Interessen hinter die Interessen des Auftragsgebers zu stellen. ${ }^{38}$ Inwieweit dies weniger ist als die fiduziarische Treuepflicht, bedarf weiterer dogmatischer Vertiefung. $\S 31$ Abs. $1 \mathrm{Nr} .1 \mathrm{WpHG}$ hat den Interessenwahrungsgrundsatz nun auch auf Geschäfte ausgedehnt, die erstmals nicht fremdnützig sind, wie etwa die Darlehensgewährung. ${ }^{39}$

Unklar ist schließlich, ob die Pflichten der Finanzintermediäre, die regelmäßig zwischen Investoren und börsennotierten Unternehmen stehen, abgeschwächt auch auf die börsennotierten Unternehmen selbst übertragen werden können. Dies wird wohl in einem ersten Schritt abzulehnen sein, weil börsennotierte Unternehmen regelmäßig nicht fremdnützig zugunsten des Anlegers agieren. Grundsätzlich fehlen vertragliche Beziehungen, so dass lediglich eine deliktsrechtliche Haftung in Betracht kommt. ${ }^{40}$ Denkbar ist es, die börsennotierten Unternehmen mit der Pflichtenstellung zu vergleichen, die bei einer Berufshaftung ${ }^{4}$ von Wirtschaftsprüfern, Steuerberatern und Rechtsanwälten bejaht wird, da auch am Kapitalmarkt auf eine besondere Sachkunde der Berufsträger vertraut wird..$^{42}$ Bei der Ad-hoc-Publizität trifft den Vorstand eines börsennotierten Unternehmens eine besondere Pflicht, weil ihm als „Informationsmonopolist" eine besondere Verantwortung zukommt. ${ }^{43}$ Die Informationen des Unternehmens beeinflussen den Börsen-

35 Hierzu etwa Möllers in Hommelhoff/Hopt/von Werder (Hrsg,), Corporate Governance-Handbuch, $2009^{2}$, S. 387.

${ }^{36}$ Ausführlich Sethe Anlegerschutz im Recht der Vermögensverwaltung, 2005, S. 149 ff., 618.

§ 22 InvG; s. auch $§ 7$ PfandBG.

ss Hopt in Baumbach/Hopt, HGB ${ }^{34}, 2010, \S 347$ Rn. 30.

Möllers in KK-WpHG, 2007, § 31 Rn. 87.

40 Zum Streitstand Möllers/Leisch in KK-WpHG, 2007, §§ 37b, c Rn. 13 ff.; Sethe in Assmann/Schneider, WpHG, §§ 37b, 37 c Rn. 18 ff. Leyens in Baum u.a., Perspektiven des Wirtschaftsrechts, 2008 , S. 423 ff.

"Hopt AcP 183 (1983) 608.

"2 Hellgardt geht von einem Sonderrechtsverhältnis aus, das sich bei Fehlinformationen zur Selbstbereicherung oder zur gezielten Ausschaltung von Corporate GovernanceMechanismen zu einer kapitalmarktrechtlichen Treuepflicht verdichten kann, Hellgardt Kapitalmarktdeliktsrecht, 2008, S. 218 ff., 436 ff.

${ }^{43}$ Hierzu erstmals Möllers/Leisch WM 2001, 1648, $1654 \mathrm{zu}$ - Infomatec; dies, in KKWpHG, 2007, §§ 37b, c Rn. 119; Möllers in Möllers/Rotter, Ad-hoc-Publizität, 2003, § 3 Rn. 32,4 7; ihnen folgend Rieckers BB 2002, 1213; Krause ZG R 2003, 799, 823. 
kurs, aber auch die Analysen Dritter. Beeinflusst diese Rolle die Pflichtenstellung und das Verschulden ${ }^{44}$ Diese Überlegungen lassen sich auch auf Jahres- und Halbjahresbilanz ausweiten, weil der Informationsgehalt im Vergleich zur Ad-hoc-Mitteilung noch deutlich hoher ist..$^{45}$ Dann müsste dies für die Pflichtenstellung erst recht gelten. Die Einführung des „Bilanzeides*** hat das Gewicht der Jahresbilanz für das Anlegerpublikum nochmals verdeutlicht.

Völlig vage sind die Anforderungen an die Jedermannspflichten des Insiderrechts und der Kurspreismanipulation. Erhöht es die Rechtswidrigkeit, wenn professionelle Marktteilnehmer den Tatbestand, etwa die Kurspreismanipulation, verwirklichen, oder hat es am Ende nur Auswirkungen auf das Strafmaß? Oder umgekehrt gefragt: Haben „Laien" einen Bonus ? ${ }^{47}$

\section{Finanzprodukte und Informationen börsennotierter Unternehmen}

\section{Komplexe Finanzprodukte - Verbot, Zulassung, Transparenz}

Banken, insbesondere Investmentbanken, sind innovativ. Während früher hauptsächlich die Aktie als Wertpapier und die Unternehmensanleihe den Markt beherrschten, sind in den letzten Jahren unzählige Finanzprodukte hinzugekommen, nicht nur um etwa Steuern zu sparen, sondern vor allem auch, um neue Märkte, neuen Handel, neuen Gewinn zu generieren. Als Beispiele seien etwa nicht nur Put- und Call-Optionen genannt, sondern auch Derivate, Short Sales, gegebenenfalls auch in der Form der Naked Short Sales oder strukturierte Produkte wie CDO oder Mortgage Backed Securities (RMBS). Das hatte zur Folge, dass komplexe Finanzprodukte vom Käufer, sei es der Kleinanleger, sei es der professionelle Anleger, nicht mehr verstanden und damit die Chancen und Risiken des Finanzproduktes nicht richtig eingeordnet wurden. Daneben haben komplexe Finanzprodukte, wie etwa

${ }^{4}$ Zum Missbrauch des Informationsinstrumentes s. Fuchs in Fuchs, WpHG 2009. vor $\S \S 37$ b, 37 cRn.34.

${ }^{45}$ Zu Recht betont der BGH, dass etwa der Jahresbilanz oder dem Börsenprospekt ein höherer Informationswert zukommt als der punktuellen Ad-hoc-Publizirat, BGH v. 19,7.2005, BGHZ 160,134, 138 - Infomatec II: Ad-hoc-Publizität im Vergleich zum Prospekt.

${ }^{46}$ § 37v Abs. 2 Nr. 3, 37w Abs. 2 Nr. 3, 37y Nr. 1 WPHG, § 264 Abs. 2 S. 3, 289 Abs. 1 S. 5 HGB. Dem steht entgegen, dass jetzt die Sanktionen ganz unterschiedlich ausfallen können, vom Straftatbestand des $\S 331$ Nr. 3a HG B bis zur Sanktionslosigkeit; s. MUlbert/ Steup NZG 2007, 761, 769; Fuchs in Fuchs, WpHG, 2009, vor $§ 37 v-37 z$ Rn, 15.

"7ibt eine Vereinigung zur Unterstützung von Anlegerinteressen ein Statement ab, existieren höhere Recherchepflichten, um Gerüchte zu vermeiden, als wenn ein Kleinanleger in Internet-Foren zu einem Wertpapier Position bezieht. 
die CDO gerade dazu geführt, dass Risiken überhaupt erst produziert wurden. So wurden Häuserkredite an letztlich nicht solvente Kunden vergeben, diese Risiken dann aber nicht in die eigenen Bücher genommen, sondern über die Märkte an Dritte weitergereicht- Drittens werden Kursbewegungen durch Wetten auf steigende oder fallende Kurse durch bestimmte Finanzprodukte noch verstärkt. Ein berühmtes BeispieHst die VW-Stammaktie, die im Herbst 2008 kurzfristig auf über 1.000 EUR stieg, dann aber wieder auf 200 EUR fiel ${ }^{48}$

Im Einzelnen wird man hier an zwei Regelungsmechanismen des Kapitalmarktrechts denken können. Einerseits muss die Diskussion starten, welche Produkte künftig verboten oder von einer Aufsichtsbehörde nur in kleinerem Umfang zugelassen werden sollten. ${ }^{49}$ Sicherlich lebt die Branche davon, ständig neue, „innovative" Finanzprodukte zu kreieren. Und Chance und Risiko stehen sich zwingend gegenüber. Investoren dürfen an den Märkten aber nur dann im weiten Umfang ,spielen", wenn sie bereit sind, ihren Einsatz zu verlieren. Deshalb sollten künftig Produkte verboten werden, die ohne Kapitalhinterlegung möglich sind und die wegen extremer Hebelwirkungen eher schädlich als dienlich sind. $§ 37 \mathrm{~g} \mathrm{WpHG}$ bildet hier einen richtigen Ansatz: Danach sollte das BMF eine Verordnung erlassen, auf deren Grundlage bestimmte Finanzprodukte verboten werden könnten. Leider wurde hiervon noch nicht Gebrauch gemacht. Richtigerweise wurden aber in den USA und in Deutschland Leerverkäufe verboten, weil hier ohne Kapitaleinsatz auf fallende oder steigende Kurse gesetzt werden kann. ${ }^{50}$ Zudem sollten künftig CDO nur dann zugelassen werden, wenn der handelnde Partner ein eigenes Risiko übernimmt. Für Privatanleger gibt es Jetzt schon Beschränkungen, etwa dass nur bei nicht-komplexen Finanzinstrumenten das Discount-Broking zulässig ist. ${ }^{51}$

Daneben muss ein Mehr an Transparenz erzeugt werden. Auf jeden Fall sollten weitergehende Informationspflichten eingeführt werden, da $\S 31$ WpHG die Gefährlichkeit von Finanztermingeschäften nicht hinreichend

${ }^{4}$ Knop F A 2 v. 14,11.2008, abrufbar unter wwwiaz.net.

${ }^{4 *}$ Ähnliche Diskussionen finden sich auch im Wettbewerbsrecht s. Fikentscher GRURInt 2009, 615; Straus/Klopschinski in FS Mes, 2009, S. 327.

so Am 15.7.2008 verbot die SEC das, ,naked short selling* von Aktien einiger Finanzinstitute. „Naked short selling* bezeichnet die Praxis, Leerverkäufe zu tätigen, ohne die Aktien vorher wirklich zu leihen oder sicherzustellen, dass sie geliehen werden können. Panikverkäufe führen nach der SEC zu einem künstlichen und unnötigen Preisverfall auf ein Preisniveau, das signifikant unter demjenigen liegt, welches sich aufgrund des normalen Preisentdeckungsverfahrens ergeben hätte, Federal Register: July 21, 2008 (Vol. 73, Nr. 140), S. 42379-42380. Auch die BaFin hat Leerverkäufe verboten und stützt sich dabei auf $\$ 4$ Abs. 1 W pHG, s. Allgemeinverfügung der BaFin v. 21.9.2008 zum Verbot von Leerverkäufen. Inzwischen will auch der Finanzminister Schäuble bestimmte Arten von Leerverkäufen verbieten.

${ }^{s 1}$ Art. 18 Abs. 6 MiFID, umgesetzt in $§ 33$ Abs. 7 WpHG. 
zum Ausdruck bringt. Die Streichung der $\S \S 37 \mathrm{~d}$ ff. WpHG, die bis zum 31.10.2007 vor besonders gefährlichen Termingeschäften warnten und vor 2002 sogar ein Schriftformerfordernis statuierten, ${ }^{52}$ ist insoweit ein Rückschritt zulasten des Anlegerschutzes. Der Kleinanleger sollte das Risiko, das er eingeht, auch wirklich überschauen. Löblich ist, dass der BGH jüngst einen Schadensersatzanspruch bejaht hat, weil nicht auf das Risiko eines Totalverlustes hingewiesen wurde. ${ }^{53}$

Hilfreich wäre noch eine stärkere Klassifizierung von Risiken. Nicht jedem Anleger ist die Gefährlichkeit des Prime Standard bewusst. Mehr Transparenz komplexer Produkte ist bei Derivaten und Zertifikaten unabdingbar. Ein Kurzinformationsblatt, das über die bisherigen Basisinformationen hinausgeht, könnte hilfreich sein. ${ }^{54}$ Besondere Risiken sollten nicht nur in einem standardisierten Formblatt ${ }^{\text {ss }}$ erwähnt werden, sondern auch im Beratungsprotokoll wieder auftauchen, um sicherzustellen, dass das spezifische Risiko auch ausdrücklich genannt wurde,

\section{Informationen börsennotierter Unternehmen und Informationsflut}

\section{a) Informationsflut}

Für börsennotierte Unternehmen gelten zahlreiche Informationspflichten. ${ }^{56}$ In den letzten Jahren wurden diese Aufklärungspflichten massiv ausgeweitet: Man erinnere nur an die Einführung und dann Ausweitung von Quartal-, Halbjahres- und Jahresbericht, Ad-hoc-Publizität, ^Director's Dealing", Beteiligungstransparenz etc. Allerdings scheinen die Grenzen der Aufklärungspflichten zunehmend erreicht zu werden, Sie belasten die Unternehmen nicht nur mit hohen Kosten, sondern führen zu Lasten des Anlegers auch $\mathrm{zu}$ einer Informationsflut («information overload"), die nicht mehr bewältigt werden kann.

s2 Zur Rechtsgeschichte s. G, Roth in KK-WpHG, 2007, § 37d Rdn, 19 ff.; Fuchs in Fuchs, WpHG, 2009, vor $\$ \$ 37$ e und 37g Rn. 92 ff,

s3 BGH v. 14.7.2009, WM 2009,1647 = ZIP 2009, 1654 Rn. 51 - BFI Bank.

${ }^{54}$ Ein vergleichbares Produktinformationsblatt gibt es bei Versicherungen, $\mathbf{s . ~} \$ 4 \mathbf{W} \mathbf{G}$ Info-V v. 18.12.2007, BGBL I, S. 2004. Zu dieser Forderung s. Studie BELV (Fn. 33), S. 62; zweifelnd dagegen Lang/Kühne WM 2009, 1301, 1303: „,eher kontraproduktiv*.

s5 Kritisch zum bisherigen Recht Casper WM 2003, 161,167 f.; Merkt Gutachten G für den 64. DJT, S. 102 f.; H.E Müller in Derlender/Knops/Bamberger Handbuch zum deutschen und europäischen Bankrecht, $2009^{2}, \S 54$ Rn. 60,

56 Für eine graphische Übersicht s. etwa Möllers in Möllers/Rotter, Ad-hoc-Publizität, 2003, § 2 Rn, 50.

Rogers/Agarwala-Roger Communications in Organisations, 1976, S，90; Jäcoby/ petler/Kohn Bertling 1 JCR 33 (1974); Malhotra 8 JCR 419 (1982); den. 10 JCR 436 (1984); für das Kapitalmarktrecht s. Langevoort 2 Entrepreneurial Bus.L J. 767, 770 (2008); Dalley 34 Fla.St.U.L.Rev. 1089 (2007); Paredes 81 Wash.UX.Q. 417, 419 (2003); Ripken 58 Baylor L.Rev. 139 (2006). 


\section{b) Bounded Rationality — Zusammenfassungen}

Hier könnten die Ergebnisse der Behavioral Finance-Forschung herangezogen werden, die auf die begrenzte Einsichtsfähigkeit des Investors abzielt $^{58}$ Lösungsansätze können etwa sein, irreführende Angaben zu vermei$\mathrm{den}^{59}$ und Informationen zu reduzieren. Gefordert wird, das Jährliche Dokument gem. $\S 10 \mathrm{WpPG}$ abzuschaffen, ${ }^{60}$ auch wenn dies wohl kaum Kosten einspart und eine Übersicht der jährlichen Informationen als Investor Relations-Maßnahme doch sinnvoll erscheint. Nachholbedarf besteht etwa noch für die Jahres- und Halb Jahresbilanzen, die wohl vom klassischen Kleinanleger nicht gelesen, geschweige denn verstanden werden. Hier bedarf es „Ubersetzungshilfen", die einfach und verständlich sind. Jetzt verpflichtet der Gesetzgeber schon zur Erstellung einer Zusammenfassung von Börsenprospekten ( $\$ 5$ Abs. $2 \mathrm{WpPG}$ ). Diese Pflicht ist auf den Sekundärmarkt zu übertragen. Künftig ist für den Jahresbericht ein Kurzfinanzbericht zu erstellen ( $(37 \mathrm{v}$ Abs. $2 \mathrm{WpHG}-\mathrm{E})$, um besser als bisher zu ermöglichen, dass diese wichtigen Unternehmensinformationen von den Anlegern überhaupt wahrgenommen werden. ${ }^{61}$ Neben den Basisinformationen könnten Wertpapierdienstleistungsunternehmen zusätzlich Kurzinformationsblätter dem Kunden bei der Anlageberatung in die Hand geben, da diese „passgenauer" den Kunden erreichen. ${ }^{62}$

ss Shiller 17 Journal of Economic Perspectives 83 (2003); Shteifer Inefficient Markets: An Introduction to Behavioral Finance, 2000, S. 10 if.|Jolls/\$unstein/Thaler 50 Stan.L.Rev. 1471,1475 (1998); Oehler Ö B A 2000, 978, 989, 978. Wegbereitend Simon 69 QJE 99 (1955); den. Administrative Behavior 1957², S. 81 f.; Miller 63 Psychology Rev. 81 (1956); Chase/ Simon 4 Cognitive Psychology 55, 76 (1973); s. auch Klöhn Kapitalmarkt, Spekulation und Behavioral Finance 2005; Schon in FS Canaris, Bd. 1, 2007, S. 1191; Stürner in FS Canaris, Bd. \}, 2007, S. 1489.

" Zu den Missständen bei der Ad-hoc-Publizität im Jahre 2000 und der Reaktion des Gesetzgebers durch die Einführung von $\$ 15$ Abs. 2 W H H s. BaFin Emittentenleitfaden 2005, S. 59; Assmann in AssmWSchneider, WpHG', 2009, § 15 Rn. 199 ff. Für Wertpapierfirmen neu durch Art. 19 Abs. 2 MiFID eingeführt etwa $§ 31$ Abs. 2 W p HG.

${ }^{6}$ So auch Götze NZG 2007, 570, 574, 570; dersJWunderlich in Habersack/Mülbert/ Schutt (Hrsg.), Handbuch der Kapitalmarktinformation, 2008, § 11 Rn. 2. Ebenso der Änderungsvorschlag zur Prospekt-RiL v. 23.9.2009, KOM (2009), 491 endg., S. 10, 5.3.9; abrufbarunterwww.thomas-moellers.de.

${ }^{61}$ In Großbritannien gibt es solche bereits seit 1995, s. Statutory Instrument 1995 No. 2092, The Companies (Summary Financial Statement) Regulations 1995. Auf freiwilliger Basis wird dies schon vorgenommen etwa von BASF SE BASF Kompakt, abrufbar unter http://berichte.basf.de/basf^^ 2008.pdf.

${ }^{62}$ S. oben F n. 54. 


\section{IV. Überwachung und Rechtsdurchsetzung}

\section{1. Öffentlich-rechtliche Aufsicht}

a) Effizienz auf europäischer Ebene

Schließlich soll noch die Überwachung genannt werden. Auch hier erlauben wir uns ineffiziente Strukturen, etwa mit 27 nationalen Aufsichtsbehörden, die oft viel zu klein sind, um international tätigen ,global playern", wie etwa den drei großen Rating-Agenturen, gleichberechtigte Partner zu sein. ${ }^{M}$

\section{b) Effizienz auf nationaler Ebene}

Bank- und Kapitalmarktrecht ist eine Gemengelage von öffentlich-rechtlichen, strafrechtlichen und zivilrechtlichen Strukturen. Die Zusammenarbeit zwischen BaFin und den Staatsanwaltschaften krankt (noch) daran, dass die Wirtschaftsdezernate der Staatsanwaltschaften fachlich und personell unterbesetzt sind. Künftig sollte die Fähigkeit der Staatsanwaltschaften, Insiderdelikte und Marktmanipulationen strafrechtlich zu verfolgen, der Bedeutung des Börsenplatzes entsprechen. Letztlich muss aber auch die Mitarbeiterzahl bei der BaFin erhöht werden. ${ }^{64}$

\section{Z Zivilrechtliche Sanktionen}

\section{a) Unzureichender Rechtszustand}

Jüngere Entwicklungen im europäischen Kartellrecht verdeutlichen, dass neben dem öffentlichen Recht auch die zivilrechtliche Rechtsdurchsetzung durch Private unabdingbar ist, um Rechtsverletzungen $\mathrm{zu}$ ahnden und eine offene Marktwirtschaft mit freiem Wettbewerb zu erzeugen, Art. 4 E G. ${ }^{65}$ Sieht man funktionierende Kapitalmärkte als ein dem funktionierenden Wettbewerb vergleichbares Rechtsgut an, bedarf es auch eines stärkeren zivilrechtlichen Rechtsschutzes, um das erforderliche Vertrauen in die Kapitalmärkte zu erzeugen. Positiv ist sicherlich die Streichung der spezialrechtlichen Verjährungsfrist des $\S 37 \mathrm{a} \mathrm{W} \mathrm{pHG}^{66}$ sowie die Einführung des Kapital-

${ }^{63}$ Kritisch Möllers JZ $2009,861,869$ f.

${ }^{64}$ Die Jahresberichte der BaFin weisen auf die noch recht überschaubare Anzahl der Fälle hin, s. etwa BaFin Jahresbericht 2006, S. 163 ff; Jahresbericht 2007, S. 172 ff; Jahresbericht 2008 , S. 154 ff, abrufbar unter: http://wwwbafin.de.

${ }^{65}$ Weißbuch über Schadensersatzklagen wegen Verletzung des EG-Wettbewerbsrechts v. 2.4.2008, KOM (2008) 165 endg., hierzu Zimmer/Logemann ZEuP 2009, 489; s. auch Möllers/Heinemann The Enforcement of Competition Law in Europe, 2007, S, 387 ff

${ }^{6} \mathrm{Zu}$ dieser Forderung Leisch in KK-WpHG, 2007, § 37a Rn. 33; Leuering/Zetsche NJW 2009,2856, 2858; Fuchs in Fuchs, WpHG, 2009, § 37a Rn. 4, 12; aA Böhm BKR 2009, 221,227; Lang/Kühne WM 2009, 1301, 1304. 
marktmusterrerfahrensgesetz (KapMuG). Ansonsten liegt allerdings noch vieles im Argen. Ein spezialgesetzlicher Anspruch besteht bisher nur für fehlerhafte Ad-hoc-Mitteilungen, Wegen hoher Anforderungen an die Kausalität läuft er nahezu leer. Anderen Informationspflichten wird üblicherweise die Schutzgesetzeigenschaft abgesprochen. ${ }^{67}$

b) Einführung weitergehender Schadensersatzansprüche

Der Gesetzgeber hatte mit dem Kapitalmarktinformationshaftungsgesetz (KapInHG) vor zwei Legislaturperioden schon einmal den Versuch der Normierung einer umfassenden Haftung für fehlerhafte Kapitalmarktinformation unternommen. Selbst wenn hier die Haftung zum Teil überzogen war, ${ }^{68}$ lässt sich der Entwurf als Ausgangspunkt für weitere gesetzgeberische Initiativen heranziehen. ${ }^{69}$ So verlangt auch europäisches Recht, Schadensersatzansprüche bei fehlerhaften kapitalmarktrechtlichen Informationen einzuführen. ${ }^{70}$ Ein reiner Innenanspruch der Gesellschaft, wie dies $\S 93$ AktG vorsieht, wird dem zum Teil nicht gerecht. Europäisches Recht ist deshalb bis heute nicht korrekt umgesetzt. ${ }^{71}$ De lege ferenda ist an die Einführung eines Schadenersatzanspruches bei fehlerhaften Quartals-, Halbjahres- und Jahresberichten zu denken. ${ }^{72}$

S. etwa die Nachweise in Hirte/Möllers (Hrsg.) KK-WpHG, 2007 zu den $§ \S 13,15$, 20a, 21 ff., 31, 32 a.E (hierzu jüngst BGH v. 19.12.2008, NJW 2008, 1734, 1735 Rn. 14 ff. = WM 2008, 825), 34b, 37v ff. WpHG.

ss S. etwa Möllers JZ 2005, 75, 79 ff. Weiterführend Hopt/Voigt in Hopt/Voigt (Hrsg.), Prospekt- und Kapitalmarktinformationshaftung: Recht und Reform in der Europäischen Union, der Schweiz und den USA, 2005, S. 9, 107 ff.; Weichen Der Anlegerschaden bei fehlerhafter Kapitalmarktpublizität, 2008; Hellgardt Kapitalmarktdeliktsrecht, 2008.

"So hatte nicht nur der DJ 2002 die Einführung gefordert; die jetzige Rechtslage wird auch in der Literatur für höchst unbefriedigend erachtet.

70 Vgl. Art. 6 Abs. 2 Prospekt-RiL 2003/71/EG v. 4.11.2003, ABI. Nr. L 345, S. 64; Art. 7, 28 Transparenz-RiL 2004/109/EG v. 15.12.2004, ABI. Nr. L 390, S. 38; Art. 50b, 50c Änderungs-RiL 2006/46/EG zur Jahresabschluss-RiL 78/660/EWG (Fn. 18).

Das gilt etwa für Art. 7 Transparenz-RiL 2004 / 1909 / E G, der lautet: „Die Mitgliedsstaaten stellen sicher, dass die Verantwortung für die in den Artikeln 4, 5, 6 und 16 vorgeschriebene Zusammenstellung und Veröffentlichung der Informationen zumindest beim Eminenten oder dessen Verwaltungs-, Leitungs- oder Aufsichtsorgan liegt und dass ihre Rechts- und Verwaltungsvorschriften über die Haftung auf die Emittenten, die in diesem Artikel genannten Organe oder die beim Emittenten verantwortlichen Personen anwendbar sind." AA Breilochs Publizität und Haftung von Aktiengesellschaften im System des europäischen Kapitalmarktrechts, 2005, S. 95.

Zum Streitstand s. etwa Möllers/Leisch in KK-WpHG, 2007, §§ 37b, 37c Rn. 19, 74; Sethe in Assmann/Schneider, WpHG, 2009, §§ 37b, 37c Rn. 24 ff.; Fuchs in Fuchs, WpHG 2009, §§ 37b, 37c Rn. 69 ff. 


\section{Gesetzgebung und Juristische Methodik im Kapitalmarktrecht}

\section{Lamfattissy-Verfahren, Vollharmonisierung und weitere Lücken}

Das Lamfalussy-Verfahren bezweckt eine Vollharmonisierung, ohne diesen Terminus zu nennen. Deshalb ist grundsätzlich von einer Vollharmonisierung durch die MiFID ${ }^{73}$, die $\mathrm{MAD}^{74}$, die Prospekt-RiL ${ }^{75}$ und die Transparenz-RiL ${ }^{76}$ auszugehen. Damit käme zwar dem EuGH die Konkretisierungskompetenz bei unbestimmten Rechtsbegriffen zu; ungeklärt ist aber die Kompetenz bei ungeklärter Regelungsintensität/ ${ }^{7}$

Das gilt etwa für die wichtige Frage, in welchem Umfang neben den Pflichten aus $\S \S 31$ ff. WpHG die Zivilgerichte ungeregelte Fragen, wie etwa nachvertragliche Beratungspflichten, ${ }^{78}$ konkretisieren können.

Zum Teil wird behauptet, der nationale Gesetzgeber sei zur Konkretisierung von nicht ausdrücklich geregelten Einzelfragen befugt. ${ }^{79} \mathrm{Nach}$ der Gegenmeinung soll bei Maximal- bzw. Vollharmonisierung der Rückgriff auf das Zivilrecht ausgeschlossen sein. ${ }^{80}$ Dem steht entgegen, dass etwa die MiFID das Zivilrecht grundsätzlich von seinem Anwendungsbereich ausnimmt. Nach einer vermittelnden Meinung wird man deshalb davon auszugehen

Für die MiFID s. Möllers in FS Horn, 2006, S. 473, 485; ders. ZEuP 2008, 480, 500; Buchmann Umsetzung vollharmonisierter Richtlinien, 2008, 95; WeicAert/Wenninger WM 2007, 627, 628; Kasten WM BKR 2007, 261, 267; Jordans WM 2007, 1827; Fuchs in Fuchs, WpHG, 2009, § 31 Rn. 254; Veil ZBB 2008, 34, 41 f.; Herresthal ZBB 2009, 348, 352; von Maximalharmonisierung spricht Mülbert WM 2007, 1149, 157; ders. ZHR 172 (2008) 170, 176.

Buche Die Pflicht zur Ad-hoc-Publizität als Baustein eines integeren Finanzmarkts, 2005, S. 85; Möllers in FS Horn, 2006, S. 473, 485; den. ZEuP 2008, 480, 500; Buchmann Umsetzung vollharmonisierter Richtlinien, 2008,100 ; ebenso Mülbert Z H R 172 (2008) 170, 181.

Für die Prospekt-RiL s. Ferran Building an EU Securities Market, 2004, 138; Buchmann Umsetzung vollharmonisierter Richtlinien, 2008, S. 100; Mülbert ZHR 172 (2008) 170,181 .

${ }^{76}$ Fleischer/Schmolke NZG 2009, 401, 408; von Maximalharmonisierung spricht Mülbert WM 2007, 1149, 1157; ders. Z H R 172 (2008) 170, 182.

"Möllers in Gsell/Herresthal (Hrsg.), Vollharmonisierung im Privatrecht, 2009, S. 247, 264 ff.

${ }^{78}$ S, oben Fn. 28.

79. etwa RegE BT-Drs- 16/12814, S. 43 zum Beratungsprotokoll sowie Voß B K R 2007, 45,48 im Rahmen von unbestimmten Rechtsbegriffen.

so Mülbert ZHR 172 (2008) 170,183.

sı S. auch Erwägungsgrund 37 der MiFID: „Von dieser Richtlinie unberührt bleiben sollte das Recht von vertraglich gebundenen Vermittlern, unter andere Richtlinien fallende Tätigkeiten und verbundene Tätigkeiten in Bezug auf Finanzdienstleistungen oder -pro $\neg$ dukte, die nicht unter diese Richtlinie fallen, auszuüben, selbst wenn dies im Namen von Teilen derselben Finanzgruppe geschieht.*. 
haben, dass die im Rahmen des Lamfalussy-Verfahrens erlassenen Richtlinien grundsätzlich aufsichtsrechtlicher Natur sind und insoweit nationale zivilrechtliche Tatbestände weiterhin anwendbar sind. ${ }^{82}$

Zur wichtigen Frage zivilrechtlicher Ansprüche sollte bei einer Änderung von MAD und MiFID Klärung auf der ersten oder der zweiten Stufe herbeigeführt werden. Denkbar wäre es, bei einer Verletzung der Pflichten aus $\S \S 31$ ff. WpHG auch einen zivilrechtlichen Schadensersatzanspruch zu begründen, wie dies etwa nach $\S 6$ Abs. 5 WG der Fall ist. ${ }^{83}$ Dann wäre zumindest dieser Standard zivilrechtlich sanktionierbar. Zudem sollte geklärt werden, in welchem Umfang die zivilrechtliche Pflichtenstellung weitergehen kann als durch die MiFID ausdrücklich geregelt. Schließlich wird die Nachfolgebehörde der CESR, die Europäische Wertpapieraufsichtsbehörde, eingerichtet, die nach Abstimmung mit der Kommission letztlich bindend technische Standards erlassen soll. ${ }^{84}$ Auch dies ist der Rechtssicherheit zuträglich.

\section{Juristische Methodik bei der Rechtsanwendung}

In der Literatur wurde der Versuch unternommen, mit dem inneren und äußeren System von Larenz ${ }^{* 5}$ eine Systematik des Kapitalmarktrechts darzustellen. Die Methodik der 60er Jahre des letzten Jahrhunderts war sicherlich hilfreich, um nach den Wirrungen des Nationalsozialismus ${ }^{86}$ zusätzliche Rechtssicherheit zu erzeugen. Allerdings scheint mir der methodische Ansatz vielleicht als „starting point" der Rechtsfindung denkbar, ansonsten aber doch aufgrund von vier Überlegungen weitgehend überholt und damit unzutreffend zu sein.

\section{a) Kapitalmarktrecht als intradisziplinäre Querschnittsmaterie}

Ganz unstrittig sind weite Teile des Kapitalmarktrechts intradisziplinäre Querschnittsgebiete, die Öffentlich-rechtlich, zivilrechtlich und strafrechtlich durchgesetzt werden. ${ }^{87}$ Bekanntermaßen sind für die einzelnen Teilrechtsgebiete aber unterschiedliche methodische Überlegungen anzustellen. Im öffentlichen Recht bedarf es einer Ermächtigungsgrundlage, wenn in die Rechtssphäre des Bürgers eingegriffen werden soll; im Strafrecht gilt der Grundsatz

${ }^{s 2}$ Koller in FS Huber, 2006, S. 821, 839 f.; Möllers WM 2008, 89, 96; ders. in Gsell/Herresthal (Hrsg,), Vollharmonisierung im Privatrecht, 2009, S. 247, 264 ff.; Veil ZBB 2008, 34, 41; aA A/ä7£ertWM 2007, 1149, U57,ders. ZHR 172(2008) 170, 183.

${ }^{83}$ Zu dieser Forderung Lang/Kühne WM 2009, 1301, 1307.

${ }^{84}$ Vorschlag für eine VO zur Einrichtung einer europäischen Wertpapieraufsichtsbehörde v. 23.9.2009, KOM (2009), 503 endg. Hierzu Möllers 11 E B O R (Heft 3); ders. NZG $2010,285$.

${ }^{s 5} \quad$ Lorenz Methodenlehre der Rechtswissenschaft' 1960; 6. und letzte Aufl. 1991.

s* Rüthers Die unbegrenzte Auslegung 2005.

${ }^{87} \mathrm{Z} / />$ te///e/>2 nc ${ }^{\wedge}$ in KK-WpHG,2007,Einl.Rn.107. 
des „nulla poena sine lege". Die zivilrechtliche Lückenschließung stößt deshalb schnell auf Grenzen. Es kann zur gespaltenen Auslegung kommen. ${ }^{88}$

b) Recht und Standardsetzung durch Aufsichtsbehörden und private Dritte

Hinzu kommt, dass weite Teile des Kapitalmarktrechts aus sog. unverbindlichem Verwaltungsinnenrecht von nationalen oder europäischen ${ }^{89}$ Finanzaufsichtsbehörden bestehen oder aus Normsetzung Privater, wie etwa der IFRS des Deutschen Rechnungslegungs-Comittee Standards (DRCS, § 342 Abs. 2 HGB) und der IDW S4 der Wirtschaftsprüfer bei der Erstellung von Börsenprospekten. Auch hier verbietet es sich, die zivilrechtliche Methodik der Auslegung von Gesetzen eins zu eins zu übernehmen. Vielmehr ist schon überaus problematisch, ob und in welchem Umfang solchen privaten Rechtssätzen überhaupt Bindungswirkung zukommt und wie dieses Recht auf staatliches Recht einwirkt. ${ }^{90}$

c) Kapitalmarktrecht als europäisches Recht

und Forschungsfeld rechtsvergleichender Studien

Deutsches Kapitalmarktrecht ist in weiten Teilen europäisches Recht. ${ }^{91}$ Das WpHG setzt beispielsweise die Marktmissbrauchs-RiL 2003/6/EG und die MiFID 2004/39/EG um, obwohl der Anwendungsbereich der Richtlinien unterschiedliche Adressaten, nämlich börsennotierte Unternehmen und Banken, betrifft. Zusammenhänge zwischen beiden Adressaten aus systematischen Gründen herzustellen, erscheint wenig überzeugend, wenn man sich vergegenwärtigt, dass sich die Marktmissbrauchs-RiL mit allen Marktteilnehmern und die MiFID hauptsächlich mit Wertpapierdienstleistungsunternehmen beschäftigt. Hinzu kommt, dass europäisches Recht vom EuGH autonom auszulegen ist und die nationalen Gerichte die Pflicht zur richtlinien $\neg$ konformen Auslegung trifft. ${ }^{92}$ Immer wieder verstoßen Gerichte gegen ihre Pflicht, Fragen zum europäischen Recht dem EuGH vorzulegen. ${ }^{93}$ Zum Teil

** S. Cohn ZHR 162 (1998) 1, 8 f.; Kalss in Riesenhuber (Hrsg.), Europäische Methodenlehre, 2006, § 20 Rn. 34; Hirte/Heinrich in KK-WpHG, 2007, Einl. Rn. 107; aA Assmann in Assmann/Schneider, WpHG, $2009^{5}$, Einl Rdn. 95.

s" Zu CESR s. Möllers ZEuP 2008,480; Spindler/Hupka in Möllers (Hrsg.) Geltung und Faktizität von Standards, 2009, S. 17; Hupka WM $2009,1351$.

" Möllers in FS Buchner, 2009, S. 649, 660 ff.; vertiefend ders. in Möllers (Hrsg.), Geltung und Faktizität von Standards, 2009, S. 143, 163 ff. Zur Auslegung der IFRS etwa Merkt in Baumbach/Hopt ${ }^{34}$, HGB, 2010 Einl. v. $\$ 238$ Rn. 135 ff

${ }^{9} \quad$ S. etwa Horn Europäisches Finanzmarktrecht, 2003.

"Hirte/Heinrich in KK-WpHG, 2007, Einl. Rn. 109; Assmann in Assmann/Schneider, WpHG, $2009^{5}$, Einl. Rdn. 96 ff.

${ }^{93}$ Beispiele für diese Vorwürfe Möllers N Z G 2008, 330, 332 zu B G H v. 25.2.2008, WM 2008,641 = NZG 2008,300 - Schremp; zu B GH v. 6.11.2003, BGHSt 48,373 - Sascha Opel; s. Vogel NStZ 2004, $252,254$. 
wird behauptet, das europäische Kapitalmarktrecht wäre grob lückenhaft und entbehre jeglichen systematischen Zugang, Gleichwohl muss aus deutscher Perspektive das Ziel weiterhin gelten, auf europäischer Ebene eine Systematisierung zu versuchen.

Die Finanzkrise hat verdeutlicht, wie global die Finanzmärkte geworden sind - deutsche Landesbanken erwarben zuhauf die giftigen Papiere US-amerikanischer Banken, mussten zwangsweise fusionieren und entgingen nur knapp dem Bankrott. Nur zu oft wurden blind US-amerikanische Rechtsregeln übernommen. Die Auslegung europäischer Richtlinien funktioniert oft nur, wenn man den Ursprung der Rechtsregeln kennt und rechtsvergleichend bei der „Mutterrechtsordnung" nach Rechtsentscheidungen suchen kann. ${ }^{94}$ Vor einer blinden Rezeption US-amerikanischen Rechts muss immer wieder gewarnt werden.

d) Kapitalmarkt und Kapitalmarktrecht-

zum interdisziplinären Charakter des Kapitalmarktrechts

Bleibt ein letzter Einwand gegen die rein zivilrechtlichen Systematisierungsversuche. Kapitalmärkte wollen effizient sein. ${ }^{95}$ Zahlreiche Modelle basieren auf ökonomischen Überlegungen. So liegt dem Kapitalmarktrecht etwa immer noch das Modell des homo oeconomicus zugrunde, also des rational handelnden Investors, der alle am Kapitalmarkt vorhandenen Informationen rational verarbeitet, so dass die Börse immer den richtigen Preis widerspiegelt. Mit diesem Modell können aber ,bubbles" ebenso wenig erklärt werden, wie die nur begrenzte Verarbeitungskapazität der Informationen. ${ }^{96}$ Erforderlich ist deshalb weniger, Lücken systematisch zu erschließen, sondern vielmehr, zuerst eine gemeinsame Sprache zwischen den verschiedenen Disziplinen zu finden ${ }^{97}$, um Thesen, die auf falschen Prämissen beruhen, mit den Mitteln des Rechts korrigieren zu können.

${ }^{94}$ Rechtsvergleichende Studien vorbildlich darstellend etwa Fleischer Gutachten F DJT 2002.

95 Möllers AcP 208 (2008) 1; s. auch Köndgen in Fleischer/Zimmer (Hrsg.), Effizienz als Regelungsziel im Handels- und Wirtschaftsrecht, Beiheft ZHR 2009, S. 100; Sester ZGR $2009,310$.

$\%$ Zu dieser Problematik s. oben III.2.

${ }^{97} \mathrm{Zu}$ dieser Forderung s. bereits Ch. Möllers in Hoffmann-Riem/Schmidt-Aßmann/ Voßkuhle, (Hrsg.), Grundlagen des Verwaltungsrechts, Bd. 1, 2006, § 3 Rn. 42: „Vorsichtsregulative*; Jestaedt Das mag in der Theorie richtig sein $2006, \mathrm{~S}$. 74: Rechtstheorie als „Grenzpostendisziplin**; zum Ruf nach einer „Meta-Dogmatik" LadeurRabelsZ 64 (2000) 60,101; Grundmann RabelsZ 61 (1997) 423. 